\title{
Aplicativo móvel para qualificar o cuidado assistencial da enfermagem aos pacientes com Diabetes mellitus tipo 1
}

Mobile application to qualify nursing care of patients with type 1 Diabetes mellitus

Aplicación móvil para calificar la atención de enfermería para pacientes con Diabetes mellitus tipo 1

Recebido: 07/12/2021 | Revisado: 15/12/2021 | Aceito: 22/12/2021 | Publicado: 04/01/2022

Carolina Piccoli

ORCID: https://orcid.org/0000-0001-5627-8376 Universidade Federal de Ciências da Saúde de Porto Alegre, Brasil E-mail: carolrpiccoli@gmail.com

Carolina Sturm Trindade

ORCID: https://orcid.org/0000-0003-3210-5360 Universidade Federal de Ciências da Saúde de Porto Alegre, Brasil E-mail: carolt@ufcspa.edu.br

Simone Travi Canabarro

ORCID: https://orcid.org/0000-0001-9339-590X Universidade Federal de Ciências da Saúde de Porto Alegre, Brasil E-mail: simonet@ufcspa.edu.br

\begin{abstract}
Resumo
Diabetes Mellitus (DM) é um crescente problema de saúde mundial. O enfermeiro realiza fortemente a educação do paciente e grupos envolvidos com a criança com Diabetes Mellitus Tipo 1 (DM1). Aplicativos móveis (Apps) para orientação no trabalho em saúde podem ser úteis como forma complementar de acesso à informação. Este estudo apresenta o processo de construção de um protótipo de um aplicativo móvel denominado DICABETES, produto de Mestrado Profissional na área de Ensino na Saúde. Trata-se de um relato de experiência escrito com base no Arco de Charles Maguerez, que fundamenta e auxilia a elaboração do pensamento crítico. Participaram na etapa de especificação de requisitos enfermeiros pediátricos. A avaliação do produto se deu através da escala de usabilidade Scale Usability Score (ScSUS), e contou com a participação de onze enfermeiros assistenciais, dois especialistas com reconhecida prática profissional em DM e três profissionais da área da Tecnologia da Informação. Os resultados indicam que, mesmo havendo necessidade de melhorias, o produto DICABETES apresentou experiência satisfatória, apresentando boa efetividade e eficiência. Assim, pretende-se aprimorar o aplicativo, a fim de disponibilizá-lo como um produto educacional para uso dos profissionais da área da saúde e para a prática diária de atendimento aos pacientes.
\end{abstract}

Palavras-chave: Diabetes mellitus tipo I; Enfermeiros pediátricos; Tecnologia em saúde; Aplicativos móveis.

\begin{abstract}
Diabetes Mellitus (DM) is a growing global health problem. The nurse is responsible for educating the patient and groups involved with the child with type 1 Diabetes Mellitus (DM1). Mobile applications (Apps) for guidance in health work can be useful as a complementary way to access information. This study presents the building process of a mobile application prototype called DICABETES, Professional Master's product in the field of Teaching in Health. This is an experience report written based on Charles Maguerez's Arc, which underpins and aids the development of critical thinking. Pediatric nurses participated in the requirements specification stage. The evaluation of the product was carried out through the Scale Usability Score (ScSUS), and it was performed by eleven treating nurses, two specialists with recognized professional practice in DM and three professionals in the area of Information Technology. The results indicate that, despite the need for improvements, the DICABETES product presented a satisfactory experience, showing good effectiveness and efficiency. Thus, it is intended to improve the application in order to make it available as an educational product for use by healthcare professionals and for the daily practice of patient care.
\end{abstract}

Keywords: Type 1 Diabetes mellitus; Pediatric nurses; Health technology; Mobile applications.

\section{Resumen}

La Diabetes Mellitus (DM) es un problema de salud mundial creciente. Los enfermeros realizan intensamente la educación de los pacientes y de grupos que incluyen a niños con Diabetes Mellitus Tipo 1 (DM1). Las aplicaciones móviles (Apps) para orientación en el trabajo en salud pueden ser útiles como forma de complementar el acceso a la información. Este estudio presenta el proceso de construcción de un prototipo de una aplicación denominada 
DICABETES, que es resultado del curso de Maestría Profesional en el área de Enseñanza en la Salud. Se trata de un relato de experiencia escrito con base en el Arco de Charles Maguerez, que fundamenta y ayuda a elaborar el pensamiento crítico. En la etapa de especificación de requisitos participaron enfermeros pediátricos. La evaluación del producto se realizó con la escala de usabilidad Scale Usability Score (ScSUS), y contó con la participación de once enfermeros asistenciales, dos especialistas con reconocida práctica profesional en DM y tres profesionales del área de Tecnología de la Información. Los resultados indican que, aunque sean necesarias algunas mejoras, el producto DICABETES presentó una experiencia satisfactoria, con un desempeño efectivo. Por lo que se pretende mejorar la aplicación, a fin de ofrecerla como un producto educacional para uso de los profesionales del área de la salud y para la práctica diaria de atención a los pacientes.

Palabras clave: Diabetes mellitus tipo 1; Enfermeros pediátricos; Tecnología para la salud; Aplicaciones móviles.

\section{Introdução}

Diabetes Mellitus (DM) é um crescente problema de saúde mundial que, independente do grau de desenvolvimento, afeta todos os países. Estima-se que 127.200 crianças e adolescentes com menos de 20 anos têm Diabetes Mellitus Tipo 1 (DM1) na Região das Américas do Sul e Central (SACA), sendo que cerca de 95.800 dessas crianças e adolescentes vivem no Brasil, fazendo com que o país ocupe a terceira posição no ranking global sobre incidência e prevalência em DM1 na faixa etária abaixo de 20 anos (IDF; SBD, 2019).

O aumento do número de casos de DM1 e o aumento do número de crianças e adolescentes internadas faz com que as famílias permaneçam mais tempo dentro do hospital. Nessas circunstâncias, a equipe de enfermagem é a que mais convive com as crianças com diabetes, atuando tanto nos aspectos referentes aos cuidados como também na educação do paciente, da família, dos amigos, das escolas e grupos envolvidos com a criança que apresente esta enfermidade (Silva, 2016). Sendo assim, no contexto das práticas em saúde, o Enfermeiro desenvolve algumas atividades essenciais, que englobam o cuidado ao paciente, a gerência do setor e de sua equipe, a educação em saúde e a pesquisa, ou seja, executa, planeja e organiza o processo de trabalho em saúde (Amorim et al., 2017).

No processo de adoecimento da criança com DM1, que integra o cuidado de enfermagem, o olhar se dirige à criança como o centro e, neste aspecto, a equipe de enfermagem deve estar apta a exercer as ações de cuidado adequadas a um bom atendimento. É necessário que o Enfermeiro esteja preparado para realização das suas atividades na administração dos medicamentos à beira do leito junto com a criança e na interação com a família. Isto significa que, além de ter de administrar corretamente as insulinas e atentar para o manejo apropriado das complicações agudas da doença, seguindo as normas preconizadas pelas diretrizes da Sociedade Brasileira de Diabetes (SBD), acredita-se que este profissional necessita manter uma relação humanizada com familiares e pacientes interagindo e criando uma relação de confiança (Medeiros et al., 2018).

O uso de tecnologias da informação e comunicação (TICS) aplicadas à área da saúde, através de dispositivos móveis, é definido pela OMS como mobile-Health ou mHealth (OMS, 2021). Em outras palavras, o mHealth pode ser definido como "práticas médicas e de saúde pública auxiliadas por aparatos portáteis, como celulares, aparelhos de monitoramento dos pacientes, assistentes pessoais digitais e outros aparelhos sem fio (Silva, Gatti, et al., 2020; OMS, 2021). A utilização de ferramentas computacionais entre os profissionais da saúde está em expansão e o potencial dessa tecnologia é reconhecido tanto por pesquisadores (Oliveira et al.; Silva, Gatti, et al.; Colicchio, 2020; Rocha et al., 2017; Abreu et al, 2018; Cordeiro et al., 2019; Tibes et al., 2014) como por organizações mundialmente respeitadas, como por exemplo a Organização das Nações Unidas (ONU) e pela OMS (Silva, Gatti, et al., 2020). Uma pesquisa conduzida pelo Comitê Gestor de Internet do Brasil sobre o uso das TICS nos estabelecimentos de saúde brasileiros aponta que, em 2019, 88\% dos médicos e 94\% dos Enfermeiros afirmaram que o uso das TICs trouxe benefícios nos processos de trabalho (Getschko, 2020).

Profissionais que fazem adequadamente o uso das TICS em saúde podem alcançar maior precisão e agilidade em seus trabalhos, e desta forma, gerar mais benefícios para os pacientes (Silva, Gatti, et al., 2020). Atualmente, há muitos aplicativos 
móveis (APPs) criados para a temática da área da saúde. A aprendizagem móvel é considerada como uma das principais tendências da aplicação no contexto educacional, visto que possibilita a ampliação das formas de ensino em saúde e atualização de conteúdos das diversas áreas (Amorim et al., 2017). No que tange à educação em saúde, permite que os profissionais se mantenham atualizados, consultem informações para atenderem situações específicas, ou mesmo realizem consultas com os pacientes.

No contexto brasileiro, embora as universidades utilizem dispositivos móveis com fins pedagógicos, por meio de acesso ao material didático dos cursos e livros eletrônicos, muitos acadêmicos desconhecem o benefício das tecnologias disponíveis como facilitadores da assistência à saúde da população (Silva, Gatti et al, 2020). Além disso, os autores enfatizam que muitas vezes os aplicativos são criados por desenvolvedores particulares, não apresentando vínculos a projetos de pesquisa e nem metodologia adequada (Silva, Gatti et al, 2020). Nessa ótica, os autores ainda salientam que "o desenvolvimento de pesquisas científicas para a produção de aplicativos móveis é importante, pois permite que os conteúdos sejam testados e analisados por profissionais com conhecimento específico das necessidades reais dos usuários. Esse fato é fundamental para planejar e implementar novas tecnologias de maneira adequada e coerente, de acordo com as demandas específicas, testadas na pesquisa e implementadas na prática." Na mesma linha, outros autores enfatizam que são "necessários mais estudos para estabelecer os possíveis papéis do uso de Apps em saúde sobre a relação ensino-aprendizado enquanto meio para maior disseminação e aplicação dessas ferramentas para a educação em saúde" (Rocha et al. 2017).

O presente estudo fez parte do trabalho de Mestrado Profissional em Ensino na Saúde de uma Enfermeira que, ao trabalhar em um centro de referência em Diabetes, constata queixas de pacientes relacionadas a demora no diagnóstico de DM1 ou a falta de esclarecimentos realmente efetivos sobre o manejo e cuidado dessa doença. Programas de Pós-graduação em Ensino na Saúde trabalham para a qualificação de recursos humanos por meio de desenvolvimento de competências que favoreçam transformações efetivas nas práticas cotidianas dos profissionais de saúde, com base em metodologias ativas de ensino- aprendizagem, problematização e/ou ensino baseado na prática e pesquisa educacional.

\section{Metodologia}

Apresenta-se um relato de experiência do processo de construção de um aplicativo móvel que visa complementar as ações de educação permanente em saúde no atendimento de pacientes com DM1. A proposta foi escrita com base no Arco de Charles Maguerez, considerando que ele promove a elaboração do pensamento crítico a respeito de um assunto e utiliza a realidade social para seu desenvolvimento, sendo constituído de cinco etapas: observação da realidade, os pontos-chaves, a teorização, as hipóteses de solução e aplicação à realidade (Prado et al., 2012).

Na Figura 1 tem-se uma representação gráfica das etapas do presente estudo, sendo descritas no texto a seguir. 
Figura 1: Planejamento e Construção do Arco de Charlez Maguerez

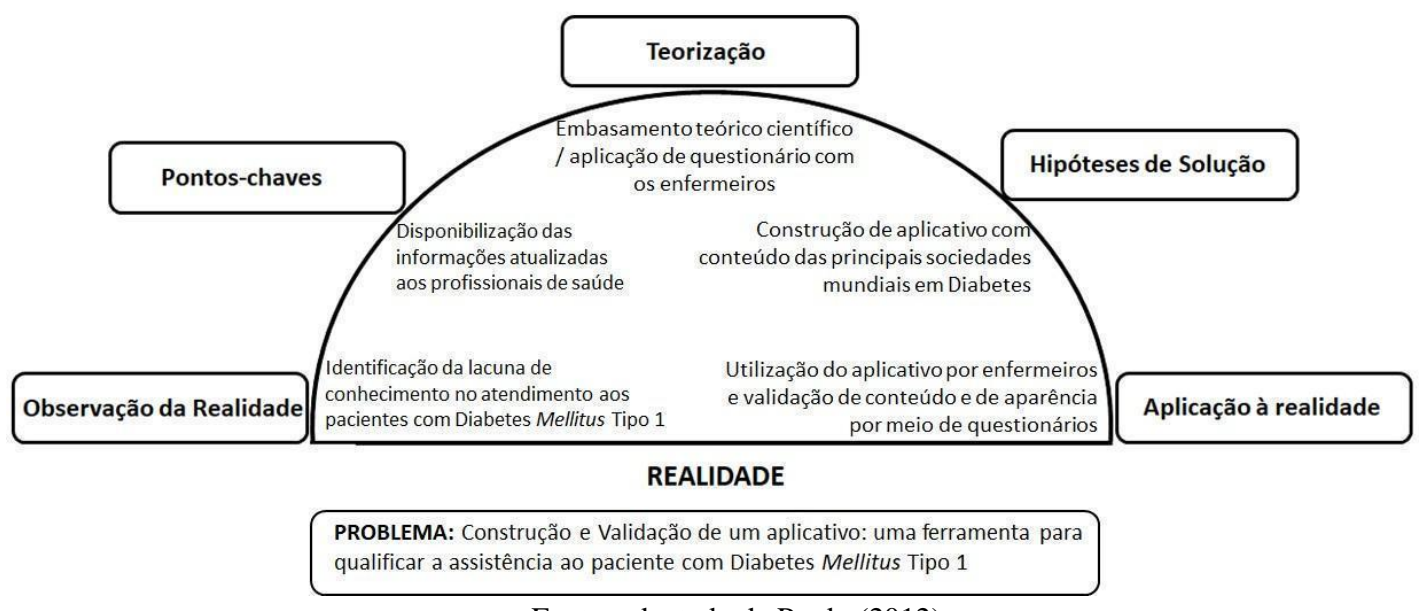

Fonte: adaptado de Prado (2012).

A Figura 1 apresenta como foram desenvolvidas as etapas deste estudo, estruturando o seu desenvolvimento.

Observando a realidade (etapa 1): Quando acontece o diagnóstico de DM1, há um impacto na vida do paciente e de sua família, são muitos os questionamentos que ocorrem durante as consultas. Surgem perguntas como "meu filho não poderá mais brincar como fazia antes?", "nunca mais meu filho poderá participar de uma festa de aniversário?", e ainda, "mas isso de usar insulina é só por um tempo, né?". E como o DM1 é uma doença autoimune onde ocorre a destruição das células beta pancreáticas, é imprescindível o uso da insulina (IDF, 2019). Diante de tantos questionamentos, preocupações, somadas ao medo das múltiplas aplicações subcutâneas, das diversas picadas no dedo para aferição da glicemia capilar, é necessário que o Enfermeiro, que irá ser o educador em saúde para eles, esclareça a maior parte dessas dúvidas, com empatia e segurança nas orientações a serem fornecidas.

O local onde ocorreu o estudo é o Instituto da Criança com Diabetes (ICD), que está vinculado a um hospital público de referência no Estado do Rio Grande do Sul (RS). A saber, o ICD é uma instituição privada, sem fins lucrativos, que desde o ano de 2004, presta assistência multidisciplinar a mais de 4.000 pacientes com (DM1) e (DM2). Foi construído através de doações de empresas e da comunidade. Há uma parceria com o Grupo Hospitalar Conceição (GHC), o qual disponibiliza os funcionários da equipe técnica multiprofissional. A Organização não Governamental (ONG) ICD realiza a captação de recursos, para poder fornecer aos pacientes insumos para seu tratamento quando o poder público falha. O Instituto disponibiliza atendimento multidisciplinar gratuito pelo Sistema Único de Saúde (SUS) para os pacientes descritos acima, através do convênio com o GHC. A equipe técnica é formada por uma equipe multidisciplinar, composta por assistentes sociais, dentistas, educador físico, endocrinologistas e endocrinologistas pediátricos, enfermeiras, nefrologista, nutricionistas, oftalmologistas, psicóloga, psiquiatra e técnicos de enfermagem (Instituto da Criança com Diabetes, 2021).

Por meio da observação do trabalho diário realizado pela equipe de enfermagem no ICD e dos relatos de familiares e cuidadores de pacientes com DM1, identificou-se que há necessidade de qualificação e capacitação referente ao atendimento e cuidados prestados aos pacientes com essa doença crônica, seja no manejo de uma situação aguda (cetoacidose diabética, hipoglicemia), bem como, nas aplicações de insulinas, realização de glicemia capilar. 
Identificando os pontos-chave (etapa 2): Nessa etapa são elencados os itens importantes a serem trabalhados, que foram identificados na observação da realidade. É aqui que ocorre a análise dos aspectos mais relevantes para serem estudados, entendidos e refletidos na busca de estratégias de melhorias (Prado et al., 2012). Os pontos identificados foram norteadores no desenvolvimento de alternativas ao tema em questão, nesse caso o desenvolvimento de uma ferramenta para que forneça suporte aos profissionais de saúde no atendimento aos pacientes com DM1.

Para identificar os pontos chave foi realizada uma consulta aos profissionais da área, ou seja, os Enfermeiros do hospital pediátrico, para identificar qual seria o conteúdo mais relevante a ser contemplado em uma estratégia de qualificação e de capacitação da equipe. A opinião destes profissionais se deu por meio de dois questionários. O primeiro foi uma adaptação do já validado Diabetes Knowledge Questionnaire (DKN-A) (Beeney et al., 2001), que é composto de 15 questões de múltipla escolha relacionados ao conhecimento geral de DM. Inclui cinco categorias: 1) fisiologia básica, o qual contempla a ação da insulina, 2) hipoglicemia, 3) grupos de alimentos e suas substituições, 4) gerenciamento de DM na intercorrência de alguma outra doença, 5) princípios gerais dos cuidados da doença. O segundo, criado pela mestranda, teve o objetivo de verificar qual o conhecimento dos Enfermeiros acerca de DM1, no que se refere às atividades com o paciente durante a internação, abrangendo as informações sobre os sinais, sintomas e principais cuidados e manejo de situações agudas no Diabetes. Após a aplicação dos questionários, foi realizada a análise das respostas dos 19 participantes e com isso se estabeleceu um caminho para a construção do conteúdo a ser disponibilizado em um aplicativo móvel, que será apresentado como um produto chamado DICABETES. Como este trabalho é fruto de um projeto de Mestrado Profissional envolvendo a participação e interação com humanos, foi submetido e aprovado pelo Comitê de Ética em Pesquisa da Universidade Federal de Ciências da Saúde de Porto Alegre (UFCSPA) e do GHC (CAAE: 35812720.1.0000.5345 / Parecer nº: 4.271.447).

Teorizando (etapa 3): Caracteriza-se pela etapa que se fundamenta o conhecimento adquirido, através das diversas fontes de informação. É nessa etapa que ocorre a descrição do conhecimento obtido com embasamento científico, contribuindo para o desenvolvimento do pensamento crítico em busca da solução de um problema. Nessa fase correlaciona-se o conhecimento prático, vivenciado, e os princípios teóricos, na busca de organizar e avaliar as informações adquiridas para saber o porquê da referida situação, compreendendo o sentido da tarefa a ser desenvolvida e a influência no contexto social (Abreu et al., 2018; Fujita et al., 2016; Villardi et al., 2015).

A seleção e elaboração do conteúdo para a construção do aplicativo foi fundamentada na análise das respostas dos questionários e na prática baseada em evidências, resultando num produto que contribui no novo cenário do uso das TICS como solução para auxiliar na rotina dos profissionais de saúde (Abreu et al., 2018; Cullum et al., 2010; Costa \& Botellho, 2020). A experiência e o conhecimento da mestranda corroboraram para sintetizar e concretizar o conteúdo a ser disponibilizado na ferramenta. Assim, a revisão de literatura abrangeu livros e artigos provenientes de bases de dados eletrônicas, versando sobre o tema DM1 bem como a construção e utilização de aplicativos móveis para a área da saúde. Também foram consideradas as diretrizes das sociedades internacionais e nacional de diabetes (Barra et al., 2018; Serafim et al., 2019).

Identificando hipóteses de solução (etapa 4): essa etapa é caracterizada pela reflexão entre teoria e realidade, estimulando a criatividade e originalidade, sugerindo atividades práticas para solução a curto, médio e longo prazo dos problemas identificados (Prado et al., 2012; Villardi et al., 2015). Considerada uma tecnologia em crescente expansão, os aplicativos desenvolvidos para dispositivos móveis apresentam a vantagem da portabilidade, ou seja, de serem facilmente disponibilizados e acessados a qualquer momento pelos seus usuários. No caso da saúde, esta característica pode ser útil como 
apoio a tratamento e promoção da assistência aos pacientes (Lima \& Barbosa, 2019; Silva, Gatti et al., 2020; Tibes et al., 2014).

Nesse contexto, foi idealizado e construído o APP denominado DICABETES. Optou-se em dispor o conteúdo de forma que ficasse prática a consulta às informações nele disponibilizadas. Na tela principal do aplicativo tem-se a divisão de seis temas, conforme ilustra a figura (2): (a) "Tela Inicial"; (b) "O que é o Diabetes"; (c) "Aplicação de Insulina - Locais e Técnica"; (d) "Insulina"; (e) "Monitorização da Glicemia"; (f) "Hipoglicemia e Cetoacidose" e (g) "Insumos". Dentro de cada item/tema abre-se uma tela com subitens dos assuntos relacionados, facilitando o acesso pelo profissional. A disposição dos temas foi pensada em uma ordem funcional, iniciando pela definição do que é o Diabetes, junto com os critérios diagnósticos e sinais e sintomas da doença. Na outra tela, aborda-se a aplicação de Insulina com os dispositivos disponíveis, técnica de aplicação e demais cuidados nessa atividade. Na sequência, a tela com o tema Insulina, onde é contada a história da Insulina, as insulinas e análogos existentes, cronologia do lançamento das insulinas e seu perfil de ação.

Em tempo, a construção do aplicativo foi realizada por um estudante da área de Tecnologia da Informação (TI), que utilizou o framework Flutter, construído pela Google, sendo testado e avaliado pela mestranda até se obter o produto final, para então ser disponibilizado para avaliação.

Nas Figuras 2, 3, 4 e 5 são ilustradas algumas telas do APP Dicabetes.

Figura 2 - tela inicial

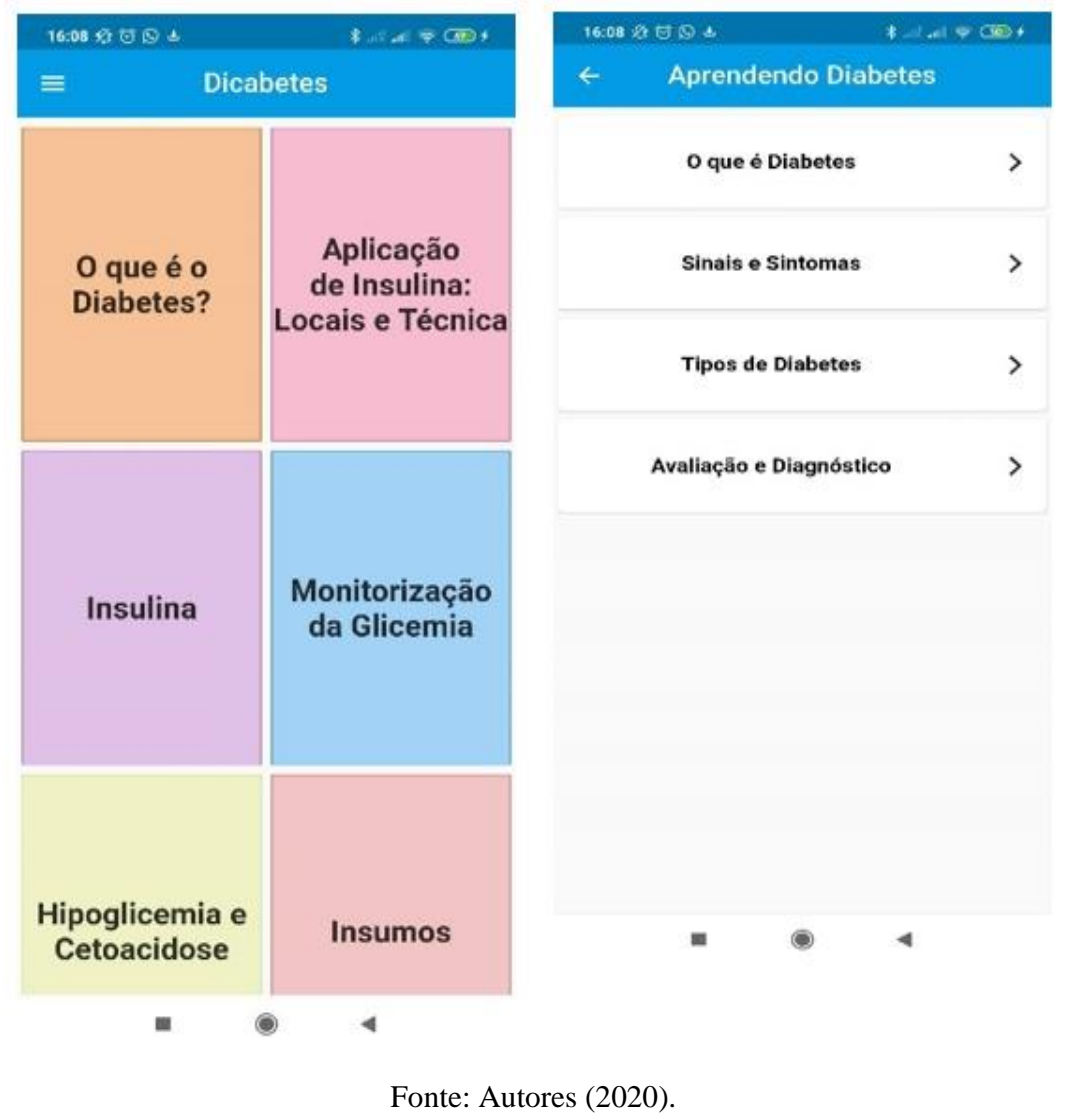


Research, Society and Development, v. 11, n. 1, e17411124477, 2022

(CC BY 4.0) | ISSN 2525-3409 | DOI: http://dx.doi.org/10.33448/rsd-v11i1.24477

Figura 4 - tela O que é o diabetes? Figura 5 - tela Sinais e Sintomas do Diabetes

$\leftarrow \quad$ Oque é Diabetes?

0 Diabetes Mellitus (DM) è uma doença crônica que ocorre quando o pâncreas não é mais capaz de produzir insulina, ou quando ○ corpo não consegue fazer bom uso da insulina que produz.

A insulina é um hormônio que age como uma chave para permitir que a glicose dos alimentos que ingerimos passe da corrente sanguinea para as células do corpo produzindo energia

Todos os alimentos com carboidratos são divididos em glicose no sangue. Não ser capaz de produzir insulina ou usá-la efetivamente eleva os niveis de glicose no sangue (conhecido como hiperglicemia).

\section{$19: 2190 \quad \$ 2100$ Sinais e Sintomas do Diabetes}

Cansaço:

Sede Excessiva;

Poliúria - em crianças menores, é comum a micção na cama:

Perda de peso; Fome excessiva;

Visão embaçada;

Mudança de humor,

Infecçōes de pele;

Dor abdominal;

- Grave (Cetoacidose diabética)

Vômitos frequentes e dor abdominal aguda; Cheiro de acetona na respiração;

Desidratação;

Diminuiçăo do nivel de consciència;

Respiraçăo Kussmaul (profunda, rápida, suspirando);

Coma;

Fonte: Autores (2020).

Figura 6 - tela Insulina

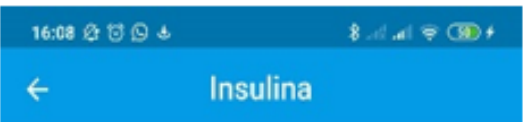

\begin{tabular}{|c|}
\hline O Advento da Insulina \\
\hline Cronologia da Insulina \\
\hline Tipos de insulina e tempo de ação > \\
\hline Insulina NPH \\
\hline
\end{tabular}

Figura 7 - tela Aplicando Insulina

16:08 \& ช (3) 4 Aplicando Insulina
$\leftarrow \quad$ A

\begin{tabular}{c} 
Dispositivos para aplicaçăo de insulina \\
\hline Técninca de aplicaçăo \\
\hline Locais de aplicaçăo de insulina \\
\hline
\end{tabular}

Fonte: Autores (2020). 
Research, Society and Development, v. 11, n. 1, e17411124477, 2022

(CC BY 4.0) | ISSN 2525-3409 | DOI: http://dx.doi.org/10.33448/rsd-v11i1.24477

Figura 8 - tela Insumos.
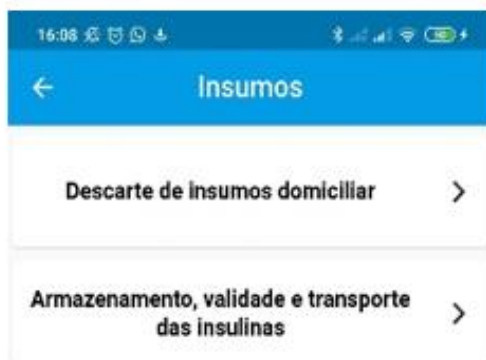

\begin{tabular}{c}
$\begin{array}{c}\text { Armazenamento, validade e transporte } \\
\text { das insulinas }\end{array}$ \\
\hline
\end{tabular}
Figura 9 - tela Armazenamento, validde e transporte das insulinas.

\section{$19: 26800$ t.48\% \\ Armazenamento, validade \\ e transporte de insulina}

As insulinas apresentam boa estabilidade $e$ têm ação preservada, se, devidamente conservadas, segundo as recomendaçōes do fabricante.

Uma forma de acompanhar a validade é anotando a data inicial de uso da insulina. Lembrando que sempre deve-se verificar o aspecto da insulina antes de sua utilização.

Frascos, refis e canetas descartáveis lacrados: manter sob refrigeração entre $2^{*}$ a $8^{\circ} \mathrm{C}$ (gaveta da geladeira).

Frascos e refis em uso: podem ser mantidos em temperatura ambiente até $30^{\circ} \mathrm{C}$, protegidos do calor e luz direta ou sob refrigeração.

Canetas descartáveis em uso: devem ser mantidos em temperatura ambiente até $30^{\circ} \mathrm{C}$, protegidos do calor e luz direta ou sob refrigeraçāo.

Frascos, refis e canetas descartáveis lacrados: validade varia de 2 a 3 anos a partir da data de fabricaçāo se armazenados sob refrigeraçāo $\left(2^{\circ}\right.$ a $\left.8^{\circ} \mathrm{C}\right)$.

Frascos, refis e canetas descartáveis em uso: validade de até 28 dias após abertos, exceto insulinas: Toujeo/Glargina $300 \mathrm{U} / \mathrm{ml}=6$ semanas (42dias) e Degludeca/Tresiba - 8 semanas (56 dias).

(SBD 2019-2020)

Fonte: Autores (2020).

Aplicação à realidade (etapa 5): nesta última etapa do Arco de Charles Maguerez acontece a implementação das soluções construídas nas etapas anteriores, contribuindo para a mudança de uma realidade. Então, após a construção do aplicativo, DICABETES, ele foi disponibilizado para que os Enfermeiros do hospital pediátrico o utilizassem na sua prática de atendimento, a fim de proceder a sua avaliação.

Considerando o crescente número de aplicativos criados para área da Saúde, faz-se necessário avaliar a usabilidade através de ferramentas específicas, para garantir a precisão e seus recursos individuais (Zahra et al., 2017). Assim, realizou-se a avaliação de usabilidade utilizando o Scale Usability Score (ScSUS). Desenvolvida em 1986, a escala numérica o ScSUS propõe a avaliação da usabilidade através de 10 questões, conforme ilustrado na figura 10, com respostas graduadas em escala Likert sendo 1 - discordo fortemente, 2 - discordo, 3 - não concordo nem discordo, 4 - concordo e 5 - concordo fortemente. $\mathrm{O}$ ScSUS ajuda a avaliar a efetividade (se os usuários conseguem completar seus objetivos); eficiência (quanto esforço e recursos são necessários para o usuário completar seus objetivos); satisfação (se a experiência foi satisfatória). 
Research, Society and Development, v. 11, n. 1, e17411124477, 2022

(CC BY 4.0) | ISSN 2525-3409 | DOI: http://dx.doi.org/10.33448/rsd-v11i1.24477

Figura 10 - Scale Usability Score (ScSUS).

\begin{tabular}{|c|c|c|c|c|c|}
\hline \multicolumn{6}{|c|}{ Pontuação } \\
\hline Questão & 1 & 2 & 3 & 4 & 5 \\
\hline $\begin{array}{l}\text { 1. Acho que gostaria de utilizar este aplicativo } \\
\text { com frequência }\end{array}$ & 0 & 0 & 1 & 6 & 4 \\
\hline $\begin{array}{l}\text { 2. Considerei o aplicativo mais complexo do } \\
\text { que o necessário }\end{array}$ & 5 & 4 & 2 & 0 & 1 \\
\hline 3. Achei o aplicativo fácil de utilizar & 0 & 0 & 0 & 6 & 5 \\
\hline $\begin{array}{l}\text { 4. Acho que necessitaria de ajuda de um } \\
\text { técnico para conseguir utilizar este aplicativo }\end{array}$ & 5 & 6 & 0 & 0 & 0 \\
\hline $\begin{array}{l}\text { 5. Considerei que as várias funcionalidades } \\
\text { deste aplicativo estavam bem integradas }\end{array}$ & 0 & 0 & 1 & 7 & 3 \\
\hline $\begin{array}{l}\text { 6. Achei que este aplicativo tinha muitas } \\
\text { inconsistências }\end{array}$ & 6 & 4 & 1 & 0 & 0 \\
\hline $\begin{array}{l}\text { 7. Suponho que a maioria das pessoas } \\
\text { aprenderia a utilizar rapidamente este } \\
\text { aplicativo }\end{array}$ & 0 & 0 & 0 & 8 & 3 \\
\hline $\begin{array}{l}\text { 8. Considerei o aplicativo muito complicado de } \\
\text { utilizar }\end{array}$ & 5 & 6 & 0 & 0 & 0 \\
\hline $\begin{array}{l}\text { 9. Senti-me muito confiante ao utilizar este } \\
\text { aplicativo }\end{array}$ & 0 & 0 & 1 & 6 & 4 \\
\hline $\begin{array}{l}\text { 10. Tive que aprender muito antes de } \\
\text { conseguir lidar com este aplicativo }\end{array}$ & 6 & 5 & 0 & 0 & 0 \\
\hline
\end{tabular}

Pontuação: (1) discordo fortemente; (2) discordo; (3) não concordo nem discordo; (4) concordo e (5) concordo fortemente.

Fonte: Autores (2020).

Ao todo, 18 profissionais avaliaram o APP utilizando o mesmo instrumento ScSUS. Primeiramente 11 usuários do DICABETES, Enfermeiros assistenciais do hospital pediátrico, realizaram a avaliação. Na etapa seguinte, cinco juizes (experts): dois membros da Sociedade Brasileira de Diabetes (com reconhecida prática profissional, currículo afim com a área avaliada e atualização permanente, considerados referências nacionais); e três profissionais da área da Tecnologia da Informação, com experiência em testes e desenvolvimento de sistemas.

\section{Resultados e Discussão}

Em um hospital geral são atendidos pacientes com os mais diversos problemas e complicações de saúde, tornando difícil para os Enfermeiros o conhecimento e peculiaridade de cada doença, e no caso do DM1 isto não é diferente. Arruda (2012) aponta que, embora sejam reconhecidas as recomendações, protocolos e diretrizes referentes ao manejo desta doença crônica, preconizadas pelas diretrizes da SBD, deve-se incentivar que o Enfermeiro busque atualização contínua com base nas demandas da sua prática diária, visando o aperfeiçoamento de competências construídas ainda na formação acadêmica (Silva, 2016). Além de desempenhar importante papel na terapêutica e recuperação, o Enfermeiro deve também intervir na prevenção. Deste modo, a equipe de enfermagem deve atuar continuamente no desenvolvimento de uma base educacional sólida aos pacientes portadores de DM1, visando o domínio de conceitos e habilidades necessários ao tratamento a longo prazo da patologia e de suas complicações (Fialho et al., 2011). 
Parte-se da compreensão de que a formação em saúde precisa estar amparada na necessária articulação com o contexto social, considerando as demandas da prática e da assistência em saúde (Silva, 2016). Para Silva, 2020, o Enfermeiro deve estabelecer uma conduta profissional baseando-se nas melhores evidências científicas, com enfoque na identificação e solução de problemas, isto é, abrangendo diagnóstico e tratamento. Logo, para que o enfermeiro possa assistir o paciente com segurança, prestando cuidado de qualidade, deve ter conhecimento sobre equipamentos, insulinas, seringas e locais de aplicação de injeção. Por fim, é recomendado a promoção de habilidades de controle do autocuidado, oportunizando aos pacientes e familiares/cuidadores a prática dos procedimentos sob sua supervisão, incluindo autoinjeção, autoteste, seleção de refeição, entre outros (Fialho et al., 2011).

A assistência ao paciente com DM1 deve estar embasada nas melhores práticas, mitigando eventos adversos. Fornecer subsídios de fácil acesso aos profissionais possibilita um atendimento com mais segurança e com maior agilidade na busca por informação. O propósito do do DICABETES é facilitar aos enfermeiros a consulta à informação correta, baseada em estudos científicos e nas diretrizes da SBD.

A SBD discorre sobre Educação em Diabetes em suas diretrizes, definindo que é processo de desenvolver habilidades para o autocuidado junto com o uso das ferramentas necessárias para atingir as metas em cada etapa do tratamento. Saliente-se que o educador em Diabetes deve identificar se o indivíduo tem condições em realizar o autocuidado, caso não possua, um familiar deverá ser capacitado para essa função de cuidados em saúde (SBD, 2019). O Enfermeiro é o profissional que está mais presente nas estratégias de educação em DM1 às crianças e adolescentes, enfatizando a importância de sua atuação no contexto educacional junto à assistência aos pacientes com essa condição crônica, independente da fase diagnóstica (La Banca et al., 2020).

A educação em saúde é um processo contínuo, que fornece ferramentas para que os profissionais de saúde reflitam suas práticas, contribuindo para diminuir os agravos à saúde. O uso dos aplicativos móveis mudou o cotidiano das pessoas e, no ensino na saúde, facilita o aprendizado dos profissionais (Costa \& Botelho, 2020; Machado et al., 2020).

A partir da construção de um APP para área da saúde, recomendações aplicáveis às esferas do ensino na assistência vem à tona, para que possam aprimorar a prática do cuidar no âmbito hospitalar, no sentido de contribuir para a redução de internações e complicações relacionadas às doenças. Quando há a construção de uma ferramenta, é necessário avaliar sua usabilidade, gerando um produto que realmente contribua para a sociedade. A usabilidade é o que avalia as facilidades de um produto, a fim de contribuir para o desempenho do design, sendo considerado um atributo de qualidade (Nielsen, 2012).

Reforça-se, então, a importância da avaliação do DICABETES feita por experts da área acadêmico-assistencial. A escolha do ScSUS se deu por não ser longo na aplicação, ser de fácil interpretação para o pesquisador, possuir qualidade, além de ser é uma ferramenta que vem sendo utilizada e referenciada na literatura (Gama \& Tavares, 2019). O ScSUS possui dois aspectos avaliativos: através das questões 1, 2, 3, 5, 6, 7, 8 e 9 considera-se a usabilidade; e as questões 4 e 10 pontuam a aprendizagem. Outro ponto importante desta escala é que ela é estruturada alternadamente entre os pontos positivos e negativos, fazendo com que os respondentes reflitam após a leitura das questões, evitando que as respostas sejam por impulso (Padrini-Andrade et al., 2019).

Com relação ao resultado do o cálculo do escore do ScSUS, constatou-se que o aplicativo DICABETES foi bem avaliado, pois dos usuários respondentes $(n=11), 45,4 \%(n=5)$ o consideraram como melhor imaginável, 36,4\% ( $n=4)$ como excelente e $18 \%(n=2)$ como bom. Dentre os juízes $(n=5), 40 \%(n=2)$ classificaram como excelente e $60 \%$ (n=3) como melhor imaginável. Quanto às considerações dos Enfermeiros, para um deles o conteúdo possui linguagem clara e fácil até mesmo para o público leigo, porém outro profissional considerou mais difícil para uma pessoa que não trabalha na área da saúde compreender. Com relação às contribuições e avaliação dos juízes especialistas, foram identificados pontos de melhoria, tais como: aperfeiçoamento do design de algumas telas do aplicativo; ajustes de conteúdo a usuários/público leigo como por 
exemplo, diminuição de conteúdo de texto e inclusão de ilustrações; ajuste de tamanho de tabelas; modificação do vídeo sobre técnica de glicemia capilar, a fim de facilitar a visualização.

Com base nas avaliações, considera-se que o aplicativo móvel DICABETES pode ser particularmente útil para a prática diária de atendimento aos pacientes, visando intervenções e inovação no ensino em saúde. Além disso, vislumbra-se que o uso do DICABETES possa ser divulgado para as diversas unidades de saúde, onde não há profissionais especialistas, ou ainda, para o público em geral, pacientes e familiares que convivem com essa condição crônica de saúde que é o Diabetes Mellitus Tipo 1.

O expressivo número de aplicativos móveis na área da saúde demonstra a aceitação do estilo de vida mais conectado, fazendo uso das facilidades de uso dessas ferramentas. Por isso, a Federação Internacional de Diabetes (International Diabetes Federation - IDF) recomenda que sejam necessários quatro níveis para o desenvolvimento de aplicativos móveis, sendo eles: individual, profissional de saúde, político e desenvolvedores de aplicativos (Rose et al., 2017). No caso do presente estudo, acredita-se que a utilização do DICABETES auxilia o trabalho dos enfermeiros, fornecendo subsídios teóricos para o desenvolvimento de sua prática diária e promoção da educação em Saúde, contribuindo para que os pacientes e familiares desenvolvam habilidades para serem os protagonistas do seu cuidado (Rosa et al., 2019; Rodrigues \& Gonçalves, 2020).

\section{Conclusão}

Acredita-se que os profissionais de saúde precisam estar atualizados para a realização de suas práticas, promovendo assistência com segurança e qualidade aos seus pacientes. Os Enfermeiros são fundamentais na promoção dos cuidados e orientações aos pacientes e familiares, contribuindo também, para a multiplicação de informações. A carga de trabalho intensa, muitas vezes em mais de um emprego, faz com que novas formas de acessar os conteúdos cooperem para sua atualização. O crescente uso de smartphones como opção de acesso aos conteúdos de educação, colabora para o desenvolvimento de aplicativos como ferramenta educativa. A metodologia usada no presente estudo, corroborou para o pensar reflexivo, na busca de alternativas para o alcance de um público maior, que possa acessar o conteúdo relacionado ao DM1. O acompanhamento criterioso do cuidado deve-se basear em práticas seguras e fundamentadas base sob a qual está construído o aplicativo que tem como referência as recomendações da Sociedade Brasileira de Diabetes.

A busca de alternativas para modificar a realidade, neste caso, nos atendimentos aos pacientes DM1, foi a motivação do presente estudo e o desenvolvimento do aplicativo DICABETES. Lança-se como desafio assistencial e acadêmico a união de esforços para sua difusão no meio hospitalar para que ocorram mudanças pragmáticas que utilizem estratégias que contemplem e assegurem o que é essencial ao cuidar a criança no mundo do hospital.

No que tange a perspectiva da pesquisa, o contexto em que ocorreu este estudo pode ser cenário de novas investigações visto seu caráter dinâmico e transformador. A utilização da tecnologia, pode ser pensada como prática educativa agregadora de conhecimento e desenvolvimento de habilidades, auxiliando no processo de ensino-aprendizagem. Além disso, fomentam a busca por novos saberes e contribuem para inovar o alcance dos recursos metodológicos de ensino. Pela importância que se atribui ao ensino, reitera-se a necessidade de um pensar e repensar conjuntamente para que o DICABETES prossiga como algo exequível e que na prática se constitua como um referencial edificando os conhecimentos do cuidador em saúde.

Faz-se necessário a continuidade na busca por conhecimento acerca do diabetes mellitus tipo 1. Sugerimos que novos estudos sejam realizados para contribuir com a prática profissional e, com isso, melhorar as condições de atendimento aos pacientes e familiares que convivem com a condição desta doença crônica. 
Research, Society and Development, v. 11, n. 1, e17411124477, 2022

(CC BY 4.0) | ISSN 2525-3409 | DOI: http://dx.doi.org/10.33448/rsd-v11i1.24477

\section{Agradecimentos}

Agradecemos ao aluno do curso de Sistema de Informação, Vinícius Hoffmann Stroff, pela colaboração e desenvolvimento do aplicativo DICABETES.

\section{Referências}

Abreu, C. A., Rosa, J. C. S., \& Matos, E. de S. (2018). Usabilidade de aplicativos móveis educacionais infantis: design e avaliação de interação do Fantastic Pirates. RENOTE, 16(1). https://doi.org/10.22456/1679-1916.86042

Amorim, L. K. de A., Souza, N. V. D. de O., Pires, A. da S., Ferreira, E. S., Souza, M. B. de, \& Vonk, A. C. R. P. (2017). O trabalho do enfermeiro: reconhecimento e valorização profissional na visão do usuário. Revista de Enfermagem UFPE on Line, 11(5), 1918-1925. https://doi.org/10.5205/1981-8963v11i5a23341p1918-1925-2017

Arruda, C., \& Silva, D. M. G. V. da. (2012). Acolhimento e vínculo na humanização do cuidado de enfermagem às pessoas com diabetes mellitus. Revista Brasileira de Enfermagem, 65(5), 758-766. https://doi.org/10.1590/s0034-71672012000500007

Barbosa, M. L. úcia K., Zemor, M. F., Zancan, L., Roesler, V., Costa, M. R. da, \& Cazella, S. C. (2018). Uma solução m-Health para apoio à educação em saúde com foco na mudança de comportamento para hábitos saudáveis. RENOTE, 16(2), 291-300. https://doi.org/10.22456/1679-1916.89269

Barra, D. C. C., Paim, S. M. S., Dal Sasso, G. T. M., \& Colla, G. W. (2018). Métodos para Desenvolvimento de Aplicativos Móveis em Saúde: revisão integrativa da literatura. Texto \& Contexto - Enfermagem, 26(4), 1-12. FapUNIFESP (SciELO). https://doi.org/https://doi.org/10.1590/010407072017002260017

Beeney, L. J., Dunn, S. M., \& Welch, G. (2001). Measurement of diabetes knowledge: the development of the DKN scales (pp. 89-159). Amsterdam: Harwood Academic Publishers.

Célia De Almeida, C., Santana, P., Naghettini, A., Tereza Vaz De Souza Freitas, A., Oliveira Barreto, G., \& Mazaro-Costa, R. (2016). Aplicativos como Estratégia de Ensino na Doença Renal Crônica Infantil: uma revisão da literatura. In Congresso Brasileiro de Informática em Saúde. https://docs.bvsalud.org/biblioref/2018/07/906273/anais_cbis_2016_artigos_completos-287-298.pdf

Colicchio, T. K. (2020). Introdução à informática em saúde: fundamentos, aplicações e lições aprendidas com a informatização do sistema de saúde americano (189). Artmed. (Original work published 2020)

Cordeiro, H. P., Silva, R. de A., Tavares, L. F., \& Bichara, C. N. C. (2019). Desenvolvimento de um aplicativo para atendimento médico de pessoas que vivem com HIV/AIDS. Revista Eletrônica Acervo Saúde, 22, e139. https://doi.org/10.25248/reas.e139.2019

Costa, L. D. A. S. da, \& Botelho, N. M. (2020). Aplicativos Móveis e a Saúde Pública Brasileira: uma revisão integrativa. Revista Conhecimento Online, 3 , 172. https://doi.org/10.25112/rco.v3i0.2144

Cullum, N., Ciliska, D., Haynes, R. B., \& Marks, S. (2010). Enfermagem Baseada em Evidências: uma introdução (1ª ed., p. 382). Artmed. (Original work published 2010)

Diabetes, I. da C. com. (2021). ICD | Instituto da Criança com Diabetes. ICD. http://www.icdrs.org.br

Diabetes, S. B. de. (2021). Diretriz da Sociedade Brasileira de Diabetes. Diretriz Da Sociedade Brasileira de Diabetes. https://diretriz.diabetes.org.br/

Federation, I. D. (2019). IDF Diabetes Atlas 9th edition 2019. Diabetesatlas.org. https://www.diabetesatlas.org

Fialho, F. A., Dias, I. M. Á. V., Nascimento, L. do, Motta, P. das N., \& Pereira, S. G. (2011). Crianças e Adolescentes com Diabetes Mellitus: Cuidados/implicações para a Enfermagem. Revista Baiana de Enfermagem25, (2). https://doi.org/10.18471/rbe.v25i2.4820

Gama, L. N., \& Tavares, C. M. de M. (2019a). Development and Evaluation of Mobile Application for the Prevention of Musculoskeletal Risks in Nursing Work. Texto \& Contexto - Enfermagem, 28. https://doi.org/10.1590/1980-265x-tce-2018-0214

Getschko, D. (2020, November 23). Resumo Executivo - Pesquisa sobre o uso das Tecnologias de Informação e Comunicação nos estabelecimentos de saúde brasileiros - TIC Saúde 2019. Cetic.br - Centro Regional Para O Desenvolvimento Da Sociedade Da Informação. https://cetic.br/pt/publicacao/resumoexecutivo-pesquisa-sobre-o-uso-das-tecnologias-de-informacao-e-comunicacao-nos-estabelecimentos-de-saude-brasileiros-tic-saude-2019/

Ghiyasvandian, S., Matourypour, P., Martins, M. M. F. P. da S., Gonçalves, M. N. da C., Ribeiro, O. M. P. L., \& Tronchin, D. M. R. (2017). Dimensioning the instrumentation: Exploratory or confirmatory factor analysis? Revista Brasileira de Enfermagem, 70(2), 233-234. https://doi.org/10.1590/0034-7167-20160183

La Banca, R. O., Sparapani, V. de C., Bueno, M., Costa, T., Carvalho, E. C. de, \& Nascimento, L. C. (2020). Strategies to Educate Young People With Type 1 Diabetes Mellitus on Insulin Therapy: systematic review. Texto \& Contexto - Enfermagem, 29. https://doi.org/10.1590/1980-265x-tce-2018-0338

Laia da Mata Fujita, J. A., Henrique de Mecena, E., Valentim Carmona, E., \& Kakuda Shimo, A. K. (2016). Uso da metodologia da problematização com o Arco de Maguerez no ensino sobre brinquedo terapêutico. Revista Portuguesa de Educação, 29(1), 229. https://doi.org/10.21814/rpe.5966

Machado, R. C. G., Turrini, R. N. T., \& Sousa, C. S. (2020). Mobile applications in surgical patient health education: an integrative review. Revista Da Escola de Enfermagem Da USP, 54. https://doi.org/10.1590/s1980-220x2018032803555 
Villardi, M. L., Cyrino, E. G., \& Berbel, N. A. N. (2015). A problematização em educação em saúde: percepções dos professores tutores e alunos (pp. 45-52). Editora Unesp. https://books.scielo.org/id/dgjm7/pdf/villardi-9788579836626-05.pdf

Medeiros, S. P., Costa, A. R., Nobre, C. M. G., Gomes, G. C., Nornberg, P. K. de O., \& Rosa, G. S. M. (2018). Percepção do familiar numa unidade pediátrica acerca do cuidado de enfermagem. Revista de Enfermagem UFPE on Line, 12(12), 3279. https://doi.org/10.5205/1981-8963-v12i12a238298p3279-3286-2018

Nielsen, J. (2012). Usability 101: Introduction to Usability. Nielsen Norman Group. https://www.nngroup.com/articles/usability-101-introduction-to-usability/

Oliveira, J. M. de Percário, S., Brito, M. V. H., Oliveira, G. C. de, Alexandre, A. D. da S., Júnior, H. S. dos S., \& Alves, M. B. M. (2020). Contribuições dos Instrumentos e Tecnologias Digitais para o Monitoramento e Controle do Diabetes Mellitus: revisão integrativa / contributions of digital instruments and technologies for monitoring and control of diabetes mellitus: integrative review. Brazilian Journal of Development, 6(12), 99564-99574. https://doi.org/10.34117/bjdv6n12-447

Padrini-Andrade, L., Balda, R. de C. X., Areco, K. C. N., Bandiera-Paiva, P., Nunes, M. do V., Marba, S. T. M., Carvalho, W. B. de, Rugolo, L. M. S. de S., Almeida, J. H. C. de, Procianoy, R. S., Duarte, J. L. M. B., Rego, M. A. S., Ferreira, D. M. de L. M., Alves Filho, N., Guinsburg, R., Diniz, E. M. de A., Santos, J. P. F. dos, Testoni, D., Silva, N. M. de M. e, \& Gonzales, M. R. C. (2019). Evaluation of Usability of a Neonatal Health Information System According to the User's Perception. Revista Paulista de Pediatria, 37(1), 90-96. https://doi.org/10.1590/1984-0462/;2019;37;1;00019

Prado, M. L. do, Velho, M. B., Espíndola, D. S., Sobrinho, S. H., \& Backes, V. M. S. (2012). Arco de Charles Maguerez: refletindo estratégias de metodologia ativa na formação de profissionais de saúde. Escola Anna Nery, 16(1), 172-177. https://doi.org/10.1590/s1414-81452012000100023

Rocha, F. S. da, Santana, E. B., Silva, É. S. da, Carvalho, J. S. M., \& Carvalho, F. L. de Q. (2017). Uso de Apps para a Promoção dos Cuidados à Saúde. Anais Do III Seminário Tecnologias Aplicadas a Educação E Saúde, III. https://www.revistas.uneb.br/index.php/staes/article/view/3832

Rodrigues, S. C., \& Schleder Gonçalves, L. (2020). Tecnologia educacional para pessoas em uso de insulina / Educational technology for people using insulin. Ciência, Cuidado E Saúde, 19. https://doi.org/10.4025/cienccuidsaude.v19i0.50376

Rosa, B. V. C. da, Girardon-Perlini, N. M. O., Gamboa, N. S. G., Nietsche, E. A., Beuter, M., \& Dalmolin, A. (2019). Development and Validation of Audiovisual Educational Technology for Families and People With Colostomy by Cancer. Texto \& Contexto - Enfermagem, 28. https://doi.org/10.1590/1980265X-TCE-2018-0053

Rose, K. J., Petrut, C., L'Heveder, R., \& de Sabata, S. (2017). IDF Europe's position on mobile applications in diabetes. Diabetes Research and Clinical Practice, 149, 39-46. https://doi.org/10.1016/j.diabres.2017.08.020

Santos Pires Lima, C., \& De Fátima Faria Barbosa, S. (2019). Aplicativos móveis em saúde: caracterização da produção científica da enfermagem brasileira. Revista Eletrônica de Enfermagem, 21. https://doi.org/10.5216/ree.v21.53278

Saúde, O. M. da. (2021, October). OMS | Brasil. WHO. http://www.who.int/eportuguese/countries/bra/pt/

Serafim, A. R. R. de M., Silva, A. N. S., Alcântara, C. M. de, \& Queiroz, M. V. O. (2019). Construção de serious games para adolescentes com diabetes mellitus tipo 1. Acta Paulista de Enfermagem, 32(4), 374-381. https://doi.org/10.1590/1982-0194201900052

Silva, J. de O. M., Santos, L. C. O., Menezes, A. N., Lopes Neto, A., Melo, L. S. de, \& Silva, F. J. C. P. da. (2020). Utilização da Prática Baseada em Evidências por Enfermeiros no Serviço Hospitalar. Cogitare Enfermagem, 26. https://doi.org/10.5380/ce.v26i0.67898

Silva, L. D. F. da. (2016). Processo de trabalho do enfermeiro no atendimento ao diabético na atenção básica (p. 27) [TCC Graduação Enfermagem]. https://bdm.unb.br/bitstream/10483/14038/6/2016_LuanaDayanneFerreiradaSilva.pdf

Silva, R. H., Gatti, M. A. N., Marta, S. N., Marafon, R. G. C., Gatti Neto, G. G., Andrade, E. B. de O. de, Andrade, S. C. de, \& Lopes, V. de C. das N. (2020).

Aplicativos de saúde para dispositivos móveis: Uma revisão integrativa / Health applications for mobile devices: An integrative review. Brazilian Journal of Health Review, 3(5), 11754-11765. https://doi.org/10.34119/bjhrv3n5-033

Tibes, C. M. dos S., Dias, J. D., \& Zem-Mascarenhas, S. H. (2014). Aplicativos móveis desenvolvidos para a área da saúde no Brasil: revisão integrativa da literatura. Revista Mineira de Enfermagem, 18(2), 471-486. https://doi.org/10.5935/1415-2762.20140035

Zahra, F., Hussain, A., \& Mohd, H. (2017). Usability evaluation of mobile applications; where do we stand? The 2nd International Conference on Applied Science and Techology 2017, II. https://doi.org/10.1063/1.5005389 